\title{
A INTERAÇÃO DE IDOSOS COM INTERFACES GRÁFICAS DE SISTEMAS DE AUTOAUTENDIMENTO: UMA REVISÃO SISTEMÁTICA SOBRE ESTE CENÁRIO
}

\section{THE INTERACTION OF ELDERLY PERSONS WITH GRAPHIC INTERFACES OF SELF-SUBSTITUTION SYSTEMS: A SYSTEMATIC REVIEW ON THIS SCENARIO}

\author{
Livia Campos ${ }^{l}$, D.Sc \\ Erika Castro $^{2}$, M.Sc \\ Luciano Tavares ${ }^{3}$, Mestrando \\ Pedro Henrique Araujo ${ }^{4}$, Graduando \\ (1) Universidade Federal do Maranhão \\ e-mail: liviaflavia@gmail.com \\ (2) Universidade Federal do Maranhão \\ e-mail:erika.ufma@gmail.com \\ (3) Universidade Federal do Maranhão \\ e-mail: raslucianot@gmail.com \\ (4) Universidade Federal do Maranhão \\ e-mail:pedrohmacedoaraujo98@gmail.com
}

Palavras-chave em português: revisão, interface, idosos.

\begin{abstract}
O presente artigo tem como objetivo apresentar considerações para aplicação de revisões sistemáticas no panorama do design, considerando as pesquisas existentes no âmbito da interação de idosos com interfaces de sistemas eletrônicos, particularmente com dispositivos de autoatendimento de caixas eletrônicos. Para tornar mais eficiente o aproveitamento do método da revisão sistemática, foi necessário organizar e resumir a informação, especificando prioridades, escolhendo fontes, categorizando de forma adequada as demandas encontradas. O presente trabalho examinou as possibilidades desse artifício como suporte na visualização do conhecimento.
\end{abstract}

Key-words in English: Revision, interface, elderly

The purpose of this article is to present considerations for the application of systematic revisions in the design landscape, considering the existing researches in the scope of the interaction of the elderly with interfaces of electronic systems, particularly with ATM self - service devices. In order to make it more efficient to use the systematic review method, it was necessary to organize and summarize the information, specifying priorities, choosing sources, and categorizing adequately the demands found. The present work examined the possibilities of this artifice as support in the visualization of knowledge. 
$16^{\circ}$ Ergodesign - Congresso Internacional de Ergonomia e Usabilidade de Interfaces Humano Tecnológica: Produto, Informações Ambientes Construídos e Transporte

$16^{\circ}$ USIHC - Congresso Internacional de Ergonomia e Usabilidade de Interfaces Humano Computador

CINAHPA | 2017 - Congresso Internacional de Ambientes Hipermídia para Aprendizagem.

\section{Introdução}

A multiplicidade do novo cenário da sociedade da informação e do conhecimento demanda uma expectativa e novos modelos, por meio dos quais a informação e o conhecimento devem ser criados, demonstrados e acessados. Com isso, é indiscutível o fato de que já estamos há tempos abarcados por informações que, segundo Wurman (1991), atuam em diferentes níveis de urgência em nossa vida.

Contudo, para a informação ser apreendida e fornecer conhecimento precisa ser organizada e resumida propiciando seu acesso e permitindo que na conjunção de seus resultados possam aparecer desfechos claros, sucintos e objetivos.

A metodologia de revisão sistemática é um esquema de revisão analítica baseada em um algoritmo explícito, que permite realizar uma pesquisa transparente e reprodutível, melhorando consideravelmente os resultados de uma revisão de literatura (GINSBERG; VENKATRAMAN, 1985; TRANFIELD; DENYER; SMART, 2003).

Classificada atualmente de grande protuberância científica, a revisão sistemática é um dos artifícios mais empregados em publicações de cunho científico de distintas áreas. Esse procedimento permite não somente a contribuição teórica, mas, sobretudo, propiciar uma vasta busca no universo das pesquisas ampliadas no âmbito nacional e internacional. Segundo Castro (2001), a revisão sistemática (sinônimos: systematic overview; overview; qualitative review) é uma revisão esquematizada para responder a uma (ou mais) pergunta específica e que utiliza métodos explícitos e sistemáticos para identificar, eleger e ponderar criticamente os estudos, e para coletar e analisar os dados destes estudos incluídos na revisão. Os processos estatísticos (meta-análise) podem ou não ser aproveitados na análise e na sinopse dos resultados dos estudos relacionados. Desse modo, a revisão sistemática emprega toda essa estruturação para evitar viés tendenciosidade. Essa ferramenta abrange a aplicação de estratégias científicas, com o escopo de restringir vieses, agrupa, avalia criticamente e resume todos os estudos importantes que respondem a um questionamento específico, além disso, promove a atualização dos profissionais e pesquisadores, uma vez que sintetiza vasto corpo de conhecimento e auxilia o esclarecimento das diferenças entre estudos com a mesma questão analítica.

\section{Estratégias adotadas na pesquisa}

Abaixo (Figura 1) observa-se o início da estrutura que evoluiu para um detalhamento maior da pesquisa, na primeira linha encontram-se as bases de dados utilizadas para filtrar os artigos necessários para construção do aporte teórico da dissertação de mestrado. Ainda na mesma linha encontra-se o tipo de documento a ser procurado, foram definidos artigos empíricos, teóricos e de revisão. A área do assunto tem relação com Arquitetura e Urbanismo e o período que interessou à coleta foram artigos de 2004 à 2016 . Na segunda linha encontra-se a quantidade de pesquisas que foram aplicadas nas bases de dados, e na linha seguinte apresentam-se as palavras chaves que foram agrupadas para realizar cada pesquisa individualmente.

É importante ressaltar a relevância desse período pois é o momento de transição do uso de terminais de autoatendimento e uso da realização de tarefas no internet banking e aplicativos móveis. Para ilustrar esta 


\section{$16^{\circ}$ \\ ERGODESIGN USIHC CINAHPA}

importância, no período de 1998 a 2004, segundo dados da Febraban (2005), as transações bancárias feitas através da Internet tiveram o notável crescimento de cerca de $100 \%$ ao ano, em média. Esse crescimento é ainda mais expressivo se comparado com outros canais: no mesmo período, as transações em caixas eletrônicos cresceram em média a $24 \%$ ao ano. A busca inicial aconteceu na revista científica Infodesign foram encontrados seis (6) artigos relacionados à pesquisa. E na última busca na revista científica Estudos em design, também obteve-se seis (6) artigos relacionados.

\subsection{Critérios de avaliação}

Abaixo segue a figura (Figura 1), que demonstra as bases de dados utilizadas, juntamente com os modelos de artigos buscados, a área do assunto, o período de seleção. A figura também demonstra que foi realizada quatro (4) buscas com quatro (4) conjuntos de palavras-chaves.

\begin{tabular}{|c|c|c|c|}
\hline $\begin{array}{l}\text { Base de dados: } \\
\text { INFODESIGN } \\
\text { ESTUDOS EM DESIGN }\end{array}$ & $\begin{array}{l}\text { Tipo de documento: } \\
\text { artigos empíricos, teóricos } \\
\text { e de revisão }\end{array}$ & $\begin{array}{l}\text { Área de assunto: } \\
\text { Arquitetura e } \\
\text { Urbanismo }\end{array}$ & $\begin{array}{l}\text { Periodo: } 2004 \\
\text { à } 2016\end{array}$ \\
\hline Pesquisa 1 & Pesquisa 2 & Pesquisa 3 & Pesquisa 4 \\
\hline $\begin{array}{l}\text { Sistema e } \\
\text { interface }\end{array}$ & Interface e idosos & Design e idosos & Sistema e design \\
\hline
\end{tabular}

A figura a seguir (Figura 2) demonstra o protocolo da revisão sistemática, o qual é composto por onze (11) intens que abrangem e caracterizam como foram desenvolvidas as buscas da revisão.

\subsection{Protocolo da revisão sistemática}

$16^{\circ}$ Ergodesign - Congresso Internacional de Ergonomia e Usabilidade de Interfaces Humano Tecnológica: Produto, Informações Ambientes Construídos e Transporte

$16^{\circ}$ USIHC - Congresso Internacional de Ergonomia e Usabilidade de Interfaces Humano Computador

CINAHPA | 2017 - Congresso Internacional de Ambientes Hipermídia para Aprendizagem.

\begin{tabular}{|c|c|}
\hline 1.Título da Pesquisa: & $\begin{array}{l}\text { A usabilidade e o usuário idoso: estudo de caso } \\
\text { da interface de autoatendimento de um banco } \\
\text { público brasileiro }\end{array}$ \\
\hline 2. Tema & $\begin{array}{l}\text { Esta pesquisa objetiva realizar a busca sistemática } \\
\text { em base de dados reconhecidas pela academia, a } \\
\text { fim de identificar estudos sobre a interaçāo dos } \\
\text { usuários idosos com dispositivos de } \\
\text { autoatendimento. }\end{array}$ \\
\hline $\begin{array}{l}\text { 3.Título da Revisão } \\
\text { Sistemática de } \\
\text { Literatura }\end{array}$ & $\begin{array}{l}\text { A interaçāo de idosos com interfaces gráficas de } \\
\text { sistemas de autoatendimento: uma revisão } \\
\text { sistemática sobre este cenário }\end{array}$ \\
\hline 4.Perguntas & $\begin{array}{l}\text { Existem padrōes de Design para desenvolvimento } \\
\text { de interfaces para sistemas de autoatendimento? } \\
\text { Como encontra-se o estado da arte em relaçāo a } \\
\text { interaçāo dos idosos com caixas eletrônicos? }\end{array}$ \\
\hline 5.Base da dados & INFODESIGN E ESTUDOS EM DESIGN \\
\hline 6.Periodo & $2004-2016$ \\
\hline 7.Palavras-chave & sistema, interface, idosos e design \\
\hline 8. Tipos de Artigos & $\begin{array}{l}\text { Serāo selecionados artigos empíricos, teóricos e } \\
\text { de revisāo. }\end{array}$ \\
\hline $\begin{array}{l}\text { 9.Critérios para } \\
\text { inclusāo }\end{array}$ & $\begin{array}{l}\text { - Artigos que tratem os aspectos de interaçāo dos } \\
\text { usuários com sistemas eletrônicos ou interativos. } \\
\text { - Artigos que apresentem estudos de cogniçāo } \\
\text { dos usuários idosos. } \\
\text { - Artigos que avaliem a usabilidade de sistemas } \\
\text { de autoatendimento. } \\
\text { - Artigos que possam conter informaçōes para } \\
\text { composição de itens que compōem uma } \\
\text { interface gráfica }\end{array}$ \\
\hline $\begin{array}{l}\text { 10.Critérios para } \\
\text { exclusão }\end{array}$ & $\begin{array}{l}\text { - Artigos que tratem da mecânica dos sistemas, } \\
\text { algoritmos, banco de dados etc. } \\
\text { - Artigos que não estejam relacionado ao tema } \\
\text { proposto }\end{array}$ \\
\hline 11.Idiomas & Português \\
\hline
\end{tabular}

Figura 2- Protocolo da revisão

O primeiro item foi o título da pesquisa que se empregado os resultados da revisão sistemática, será o desenvolvimento teórico de uma pesquisa de mestrado intitulada " $\mathrm{A}$ usabilidade e o usuário idoso: estudo de caso da interface de autoatendimento de um banco público brasileiro. O tema associa-se à realizar a busca sistemática em base de dados reconhecidas pela academia, a fim de identificar estudos sobre a interação dos usuários idosos com dispositivos de 
autoatendimento. O título da pesquisa deste artigo denomina-se "A interação de idosos com interfaces gráficas de sistemas de autoatendimento: uma revisão sistemática sobre este cenário", este título tem relação direta e indireta com o estado da arte que abrange os temas relacionados às palavras chaves (sistema, interface, idosos e design.)

As perguntas da busca da revisão sistemática são: Existem padrões de Design para desenvolvimento de interfaces para sistemas de autoatendimento? Como encontra-se o estado da arte em relação a interação dos idosos com caixas eletrônicos?, enquanto que para encontrar as respostas para essas perguntas, foi nescessário realizar as buscas em bases de dados específicas como INFODESIGN e ESTUDOS EM DESIGN. O período de busca dos artigos foi definido entre os anos de 2004 à 2016. Os tipos de artigos utilizados nas buscas foram de seleção empírica, teórica e de revisão. Os critérios de inclusão foram:

- Artigos que tratem os aspectos de interação dos usuários com sistemas eletrônicos ou interativos.

- Artigos que apresentem estudos de cognição dos usuários idosos.

- Artigos que avaliem a usabilidade de sistemas de autoatendimento.

- Artigos que possam conter informações para composição de itens que compõem uma interface gráfica

O idioma utilizado nas buscas foi o português e os critérios para exclusão foram:

- Artigos que tratem da mecânica dos sistemas, algoritmos, banco de dados etc. $16^{\circ}$ Ergodesign - Congresso Internacional de Ergonomia e Usabilidade de Interfaces Humano Tecnológica: Produto, Informações Ambientes Construídos e Transporte

$16^{\circ}$ USIHC - Congresso Internacional de Ergonomia e Usabilidade de Interfaces Humano Computador

CINAHPA | 2017 - Congresso Internacional de Ambientes Hipermídia para Aprendizagem.

- Artigos que não estejam relacionado ao tema proposto

\subsection{Conjunto de coleta das bases de dados}

O processo da coleta dos artigos originou-se a partir de buscas em cada base de dados feito de maneira individual dividindo-se em três etapas conforme citado no começo do capítulo (o planejamento da revisão, a condução da revisão e a escrita da revisão). $\mathrm{Na}$ etapa do planejamento da revisão, foram feitos estudos de viabilidade, foram definidas as perguntas da pesquisa, foi elaborado e validado o protocolo de revisão conforme a Figura (3) acima, no momento da condução da revisão buscou-se estudos primários (coleta dos artigos), foram selecionados os estudos primários relacionados com a pesquisa, a qualidade desses estudos deu-se a partir da observação e análise dos resumos de cada busca nas bases de dados. Ainda nessa etapa concluiuse a extração e e síntese dos resultados a partir da oferta da qualidade dos resumos. E neste tópico está concretizando-se a última etapa, onde se está escrevendo e validando o documento.

\section{Resultados}

\subsection{Conjunto de resultados INFODESIGN}

Neste conjunto de seleção, para o primeiro grupo (sistema e interface), houve uma busca onde encontrou-se quatro (4) artigos, onde desse grupo subtraiu-se os quatro (4) por não ter relação com a pesquisa, totalizando zero (0) artigos deste grupo aproveitado. Para o segundo grupo (interface e idosos), foram encontrados trinta e três (33) artigos, subtraiu-se vinte e nove (29) deste total, e obteve-se como resultado
Realização:

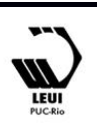




\section{$16^{\circ}$ \\ ERGODESIGN USIHC CINAHPA}

apenas quatro (4) artigos. No terceiro grupo (design e idosos), encontrou-se apenas um (1) artigo que foi empregado na pesquisa. No último grupo (sistema e design), foram encontrados vinte e um (21) artigos, dos quais eliminaram-se vinte (20) do total e apenas um (1) foi aproveitado. Dos artigos empíricos, teóricos e de revisão, encontrados ao total sendo cinquenta e nove (59) artigos, foram aproveitados para o aporte teórico um número de seis (6) artigos dos quais embasaram o referencial teórico como suporte na parte de design informacional de interfaces e também contribuiu no âmbito da cognição dos idosos.

Abaixo o quadro dos resultados obtidos na revista Infodesign, onde seguem a quantidade de artigos obtidos, os títulos dos artigos relacionados com a pesquisa, juntamente com a correspondência da edição, autoria e ano da publicação.

\begin{tabular}{|c|c|c|c|}
\hline \multicolumn{4}{|c|}{ Resultados da Revista Infodesign } \\
\hline Item & Título & Edição/ Autor & Ano \\
\hline 1 & $\begin{array}{l}\text { A contribuição da psicologia cognitiva } \\
\text { para o design de instruções de } \\
\text { procedimentos cognitivos, } \\
\text { recomendações do design, instruções } \\
\text { de procedimentos. }\end{array}$ & $\begin{array}{l}\text { v.1,n.1 (2004) } \\
\text { Franck Ganier }\end{array}$ & 2004 \\
\hline 2 & $\begin{array}{l}\text { Design de interface sistematizado como } \\
\text { processo de produto. }\end{array}$ & $\begin{array}{l}\text { v.9,n.3 (2012) } \\
\text { Jaire Ederson Passos, } \\
\text { Tânia Luisa Koltermann } \\
\text { da Silva }\end{array}$ & 2012 \\
\hline 3 & $\begin{array}{l}\text { Questões complexas do design da } \\
\text { informação e de interação }\end{array}$ & $\begin{array}{l}\text { v.7,n.2 (2010) } \\
\text { Cristina Portugal }\end{array}$ & 2010 \\
\hline 4 & $\begin{array}{l}\text { Ergodesign e arquitetura da informação: } \\
\text { trabalhando com o usuário }\end{array}$ & $\begin{array}{l}\text { v. } 4, \text { n. } 2(2007) \\
\text { Renata Zilse }\end{array}$ & 2007 \\
\hline 5 & InfoDesign-Design da Informação & $\begin{array}{l}\text { v.1,n.1 (2004) } \\
\text { Carla Spinillo, Priscila } \\
\text { Farias. }\end{array}$ & 2004 \\
\hline 6 & $\begin{array}{l}\text { Evolução das variáveis teórico- } \\
\text { empíricas interdisciplinares associadas } \\
\text { ao design no contexto do } \\
\text { desenvolvimento de interfaces para } \\
\text { ambientes virtuais de aprendizagem }\end{array}$ & $\begin{array}{l}\text { v.13,n.2 (2016) } \\
\text { Luana Maia Conti, } \\
\text { Patrícia de Sá Freire, } \\
\text { Simone Meister } \\
\text { Bilessimo, Solange Silva }\end{array}$ & 2016 \\
\hline
\end{tabular}

Quadro 1 - resultados revista INFODESIGN

\subsection{Conjunto de resultados ESTUDOS EM DESIGN}

$16^{\circ}$ Ergodesign - Congresso Internacional de Ergonomia e Usabilidade de Interfaces Humano Tecnológica: Produto, Informações Ambientes Construídos e Transporte

$16^{\circ}$ USIHC - Congresso Internacional de Ergonomia e Usabilidade de Interfaces Humano Computador

CINAHPA | 2017 - Congresso Internacional de Ambientes Hipermídia para Aprendizagem.
Nas buscas encontradas na última base de dados, encontrou-se no primeiro grupo de palavras (sistema e interface), apenas um (1) artigo que não foi aproveitado, totalizando zero (0) selecionados. No segundo grupo (interface e idosos), foram encontrados treze (13) artigos, eliminou-se oito (8) do total, e cinco (5) foram empregados na pesquisa. Ao realizar as buscas do terceiro grupo (design e idosos), achou-se três (3) artigos, eliminou-se dois (2) e aproveitou-se um (1) artigo. Para o último grupo (sistema e design), encontrou- se dezesseis (16) artigos onde nenhum foi aproveitado, totalizando zero (0) de aproveitamento deste grupo para $\mathrm{o}$ aporte teórico. Desta forma, encontrou-se nesta base de dados trinta e três (33) artigos, dos quais apenas seis (6), foram empregados na pesquisa para fundamentar os capítulos de referenciais teóricos, o conteúdo encontrado nos resultados nesta base de dados fundamentaram $\mathrm{o}$ capítulo referente à interação dos idosos com dispositivos. Abaixo o quadro com os correspondentes resultados da revista Estudos em Design.

\begin{tabular}{|c|c|c|c|}
\hline \multicolumn{4}{|c|}{ Resultados da Revista Estudos em Design } \\
\hline Item & Título & Edição/ Autor & Ano \\
\hline 1 & $\begin{array}{l}\text { Terceira idade, design universal e aging- } \\
\text { in- place }\end{array}$ & $\begin{array}{l}\text { v.24,n.1 (2016)Camila } \\
\text { Feldberg Porto, Edson } \\
\text { José Carpintero Rezende }\end{array}$ & 2016 \\
\hline \multirow{2}{*}{2} & \multirow{2}{*}{$\begin{array}{l}\text { Análise ergonômica da situação dos } \\
\text { idosos pedestres em relação à } \\
\text { sinalização de Copacabana. }\end{array}$} & v.17,n.2 (2009) & \\
\hline & & $\begin{array}{l}\text { Lianad'Urso de Souza } \\
\text { Mendes, Anamaria de } \\
\text { Moraes }\end{array}$ & 2009 \\
\hline 3 & $\begin{array}{l}\text { Dificuldades e estratégias no uso de } \\
\text { múltiplos medicamentos por idosos no } \\
\text { contexto do design da informação }\end{array}$ & $\begin{array}{l}\text { v.24,n.3 (2016) } \\
\text { Cláudio Henrique da Silva, } \\
\text { Carla Galvão Spinillo }\end{array}$ & 2016 \\
\hline 4 & Design e as interfaces de serviço & $\begin{array}{l}\text { v.23,n.1 (2015) } \\
\text { Fernando Secomandi }\end{array}$ & 2015 \\
\hline 5 & $\begin{array}{l}\text { Usabilidade de Produtos de consumo: } \\
\text { uma análise dos conceitos, métodos e } \\
\text { aplicações }\end{array}$ & $\begin{array}{l}\text { v.21,n.2 (2013) } \\
\text { Christianne Falcão }\end{array}$ & 2013 \\
\hline 6 & $\begin{array}{l}\text { Design de interfaces em software } \\
\text { educacionais: algumas recomendações } \\
\text { a partir da aplicação do método Co- } \\
\text { descoberta }\end{array}$ & $\begin{array}{l}\text { v.13,n.2 (2016) } \\
\text { Luana Maia Conti, Patrícia } \\
\text { de Sá Freire, Simone Meister } \\
\text { Bilessimo, Solange Silva }\end{array}$ & 2016 \\
\hline
\end{tabular}

Realização:

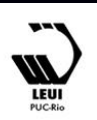




\section{$16^{\circ}$ \\ ERGODESIGN \\ USIHC CINAHPA}

Quadro 2 - resultados revista ESTUDOS EM DESIGN

Ao total utilizou-se na pesquisa seis (6) artigos relacionados à pesquisa, onde foi possível ter aporte para construir referencial teórico para estruturar a fundamentação teórica da pesquisa de mestrado. A fundamentação classificou-se em "Estudos de surgimento e evolução dos terminais de autoatendimento", "Estudos sobre a cognição e percepção dos idosos", "Estudos relacionados à usabilidade e por fim, "Estudos sobre design de interfaces".

\section{Considerações finais}

A relevância da revisão sistemática de literatura para o desenvolvimento da pesquisa relaciona-se ao fato da grande e crescente quantidade de informações que devem ser reunidas, organizadas, avaliadas, e, se possível, mensuradas para possibilitar a elaboração de conhecimento. Trata-se de uma procedimento científico muito rico para direcionar trabalhos acadêmicos, uma vez que pondera pesquisas e teorizações de distintos e múltiplos autores. Os resultados desta pesquisa sistemática foi de grande importância para construção teórica da pesquisa de mestrado em questão.

\section{Referências}

CASTRO, A. A. Revisão sistemática e MetaAnálise. p. 2-11, 2001. Disponível em: $\langle$ http://www.metodologia.org $>$. Acesso em: 28 feveireiro 2017.

DE LIMA, M. S.; SOARES, B. G.;

BACALTCHUK, J. Psiquiatria baseada em evidências. Revista Brasileira de Psiquiatria, v. 22, n. 3, p. 142-146, 2000.

FEBRABAN - Federação Brasileira de Bancos. Dados do setor: Tecnologia. $16^{\circ}$ Ergodesign - Congresso Internacional de Ergonomia e Usabilidade de Interfaces Humano Tecnológica: Produto, Informações Ambientes Construídos e Transporte

$16^{\circ}$ USIHC - Congresso Internacional de Ergonomia e Usabilidade de Interfaces Humano Computador

CINAHPA | 2017 - Congresso Internacional de Ambientes Hipermídia para Aprendizagem.

\section{Disponível}

em<http://www.febraban.org.br/Arquivo/Servico $\mathrm{s} /$

Dadosdosetor/tecnologia_2005_dadossetor.as p>. Acesso em 2017.

GINSBERG, A.; VENKATRAMAN, N. "Contingency perspective of organizational strategy: a critical review of the empirical research". Academy of Management Review, 10, 421-34, 1985.

HAMER, Susan; COLLINSON, Gill. Achieving evidence-based practice: $A$ handbook for practitioners. Elsevier Health Sciences, 2014.

PERISSÉ, André RS et al. Revisões sistemáticas (inclusive metanálises) e diretrizesclínicas. Gomes $\mathrm{M}$ da $\mathrm{M}$, organizador. Medicina baseada em evidências: princípios e práticas. Rio de Janeiro (RJ): Reichmann \& Affonso, p. 13148, 2001

TRANFIELD, D.; DENYER, D.; SMART, P. "Towards a methodology for developing evidence-informed management knowledge by means of systematic review". British Journal of Management, 14, 207-22, 2003.

WURMAN, Richard Saul. Ansiedade de informação: como transformar informação em compreensão. São Paulo: Cultura Editores Associados, 2003. 380 p. ISBN 85293-008-4. 